\title{
Numerical Modeling of the Motion of the Flat Textile Structures
}

\author{
Piotr Szablewski \\ Department of Technical Mechanics and Informatics, Technical University of Lodz, Ulica Zeromskiego 116, 90924 Lodz, Poland
}

Correspondence should be addressed to Piotr Szablewski, piotr.szablewski@p.lodz.pl

Received 22 January 2011; Accepted 24 March 2011

Academic Editor: W.-H. Hsieh

Copyright (C) 2011 Piotr Szablewski. This is an open access article distributed under the Creative Commons Attribution License, which permits unrestricted use, distribution, and reproduction in any medium, provided the original work is properly cited.

In many problems from the field of textile engineering (e.g., fabric folding, motion of the sewing thread) it is necessary to investigate the motion of the objects in dynamic conditions, taking into consideration the influence of the forces of inertia and changing in the time boundary conditions. This paper deals with the model analysis of the motion of the flat textile structure using Lagrange's equations in two variants: without constraints and with constraints. The motion of the objects is under the influence of the gravity force. Lagrange's equations have been used for discrete model of the structure.

\section{Introduction}

In most cases of dynamic analysis of textile structures, it is necessary to use the model of heave elastica, as a onedimensional body of linear weight $q$ and bending rigidity $C$ in the state of large deflections. In this paper, it is assumed that during the run of bending effect the flat textile structure (e.g., fabric) will be represented as its longitudinal section. The mathematical model will be described as a flat deflection curve; this will be treated as a heavy elastica, as shown in Figure 1 and in [1].

The word "heavy" underlines the decisive influence of the gravity forces during the motion. It is assumed that the particular longitudinal sections do not act on each other by internal forces. Furthermore, the constancy of properties along the whole width of the bending strip is assumed. It will be assumed also that the elastica is inextensible. The analysis was made using Lagrange's equations, describing the motion of the system of $n$ particles in conservative force field. Two variants of Lagrange's equations were considered: equations without constraints and equations with constraints.

\section{Discrete Model of the Object and the Lagrange's Equations}

The Lagrange's equations, describing the motion of the system of $n$ particles in conservative force field, studied in detail among other things in [2-5], are presented below:

$$
\frac{d}{d t}\left(\frac{\partial T}{\partial \dot{\varphi}_{k}}\right)-\frac{\partial T}{\partial \varphi_{k}}+\frac{\partial U}{\partial \varphi_{k}}=0, \quad k=1,2, \ldots, n,
$$

where $T$-kinetic energy, $U$-potential energy, $\varphi_{k}, \dot{\varphi}_{k} k$ th generalized coordinate and velocity. If we have $n$ generalized coordinates, then we can write $n$ Lagrange's equations (1). The problem reduces to appropriate choosing of generalized coordinates $\varphi_{k}$, deriving the formula for kinetic and potential energy, and next deriving and solving the Lagrange's equations.

During the analysis, we replace often continuous systems by discrete systems using partition into $n$ elements. In considered problem partition was made as follows. The elastica of length $L$, mass $M$ and bending rigidity $C$ fixed at point $\mathrm{A}$ was replaced by system of $n$ masses of identical value $m=M / n$, connected by $n$ straight, inextensible, and weightless segments of length $l=L / n$, as shown in Figure 2 and work [6]. The other end B of elastica is free. If $\rho$ is linear mass of elastica, then $M=\rho L$. The position of $i$ th mass is described by generalized coordinate as an inclination angle $\varphi_{i}(i=1,2, \ldots, n)$ of $i$ th segment to the vertical 


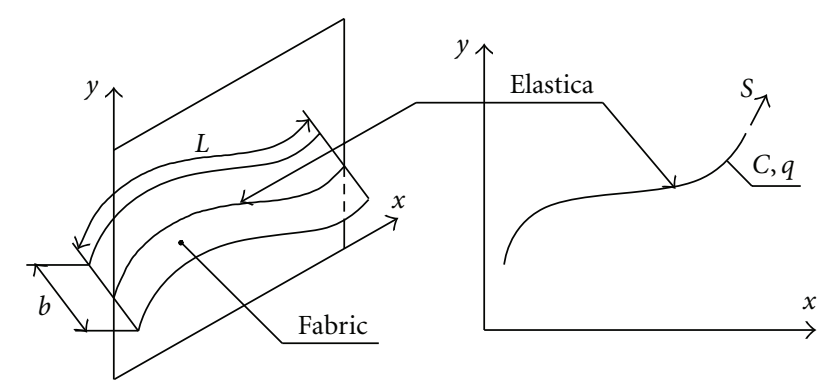

Figure 1: The model of fabric approximate to elastica.

direction. Coordinates of $i$ th mass and its derivatives are as below:

$$
\begin{aligned}
x_{i}=l \sum_{j=1}^{i} \sin \varphi_{j}, & \dot{x}_{i}=l \sum_{j=1}^{i} \cos \varphi_{j} \cdot \dot{\varphi}_{j}, \\
y_{i}=l \sum_{j=1}^{i} \cos \varphi_{j}, & \dot{y}_{i}=-l \sum_{j=1}^{i} \sin \varphi_{j} \cdot \dot{\varphi}_{j} .
\end{aligned}
$$

The kinetic energy of $i$ th mass is $T_{i}=1 / 2 m\left(\dot{x}_{i}^{2}+\dot{y}_{i}^{2}\right)$. is

Therefore, the total kinetic energy of the system of $n$ mass

$$
T=\frac{1}{2} m l^{2} \sum_{i=1}^{n}\left[\left(\sum_{j=1}^{i} \cos \varphi_{j} \cdot \varphi_{j}\right)^{2}+\left(\sum_{j=1}^{i} \sin \varphi_{j} \cdot \varphi_{j}\right)^{2}\right] .
$$

The total potential energy of the system $U$ is the sum of two components

$$
U=V+V_{b},
$$

where $V$ is the potential energy of gravity forces and $V_{b}$ is the potential energy of the bending.

The potential energy of gravity forces is $V=$ $\sum_{i=1}^{n} m g y_{i}=m g l \sum_{i=1}^{n} \sum_{j=1}^{i} \cos \varphi_{j}$ and the potential energy of the bending $V_{b}=1 / 2 C \int_{0}^{L}(d \varphi / d s)^{2} d s$. After substitution the derivative by difference quotient, we have

$$
V_{b}=\frac{1}{2} C \sum_{i=1}^{n-1}\left(\frac{\varphi_{i+1}-\varphi_{i}}{l}\right)^{2} l=\frac{C}{2 l} \sum_{i=1}^{n-1}\left(\varphi_{i+1}-\varphi_{i}\right)^{2} .
$$

Thus, the total potential energy of the system $U$ is

$$
U=V+V_{b}=m g l \sum_{i=1}^{n} \sum_{j=1}^{i} \cos \varphi_{j}+\frac{C}{2 l} \sum_{i=1}^{n-1}\left(\varphi_{i+1}-\varphi_{i}\right)^{2} .
$$

The formula for derivative of kinetic energy $T$ in respect of $\varphi_{k}$ after transformations is as follows:

$$
\frac{\partial T}{\partial \varphi_{k}}= \begin{cases}m l^{2} \sum_{i=1}^{n} \sum_{j=1}^{i} \sin \left(\varphi_{j}-\varphi_{k}\right) \cdot \dot{\varphi}_{j} \dot{\varphi}_{k}, & \text { for } i \geq k, \\ 0, & \text { for } i<k .\end{cases}
$$

After changing the limits of summation, we can write

$$
\frac{\partial T}{\partial \varphi_{k}}=m l^{2} \sum_{i=k}^{n} \sum_{j=1}^{i} \sin \left(\varphi_{j}-\varphi_{k}\right) \cdot \dot{\varphi}_{j} \dot{\varphi}_{k}
$$

Similarly, the other derivatives can be written as follows:

$$
\begin{gathered}
\frac{d}{d t}\left(\frac{\partial T}{\partial \dot{\varphi}_{k}}\right) \\
=m l^{2} \sum_{i=k}^{n} \sum_{j=1}^{i}\left[\cos \left(\varphi_{j}-\varphi_{k}\right) \cdot \ddot{\varphi}_{j}\right. \\
\left.\quad-\sin \left(\varphi_{j}-\varphi_{k}\right) \cdot\left(\dot{\varphi}_{j}-\dot{\varphi}_{k}\right) \dot{\varphi}_{j}\right], \\
\frac{\partial V}{\partial \varphi_{k}}=-m g l \sum_{i=k}^{n} \sin \varphi_{k}=-(n-k+1) m g l \sin \varphi_{k}, \\
\frac{\partial V_{\mathrm{b}}}{\partial \varphi_{k}}=\frac{C}{l} \sum_{i=1}^{n-1}\left[\left(\varphi_{i+1}-\varphi_{i}\right) \cdot \frac{\partial}{\partial \varphi_{k}}\left(\varphi_{i+1}-\varphi_{i}\right)\right] .
\end{gathered}
$$

The derivative $\partial\left(\varphi_{i+1}-\varphi_{j}\right) / \partial \varphi_{k}$ depending on the value of index $i$ is given by

$$
\frac{\partial}{\partial \varphi_{k}}\left(\varphi_{i+1}-\varphi_{i}\right)= \begin{cases}1, & \text { for } i=k-1, \\ -1, & \text { for } i=k \\ 0, & \text { for } i<k-1, i>k\end{cases}
$$

Using summation from $i=1$ to $i=n-1$ the formula (11) can be written as follows:

$$
\frac{\partial V_{b}}{\partial \varphi_{k}}= \begin{cases}\frac{C}{l}\left(\varphi_{1}-\varphi_{2}\right), & \text { for } k=1, \\ \frac{C}{l}\left(-\varphi_{k-1}+2 \varphi_{k}-\varphi_{k+1}\right), & \text { for } k=2,3, \ldots, n-1, \\ \frac{C}{l}\left(\varphi_{n}-\varphi_{n-1}\right), & \text { for } k=n .\end{cases}
$$

Therefore, the derivative of total potential energy in respect of $\varphi_{k}$ is

$$
\begin{aligned}
\frac{\partial U}{\partial \varphi_{k}}= & \frac{\partial V}{\partial \varphi_{k}}+\frac{\partial V_{z g}}{\partial \varphi_{k}} \\
= & -m g l \sum_{i=k}^{n} \sin \varphi_{k} \\
& +\frac{C}{l} \sum_{i=1}^{n-1}\left[\left(\varphi_{i+1}-\varphi_{i}\right) \cdot \frac{\partial}{\partial \varphi_{k}}\left(\varphi_{i+1}-\varphi_{i}\right)\right] .
\end{aligned}
$$




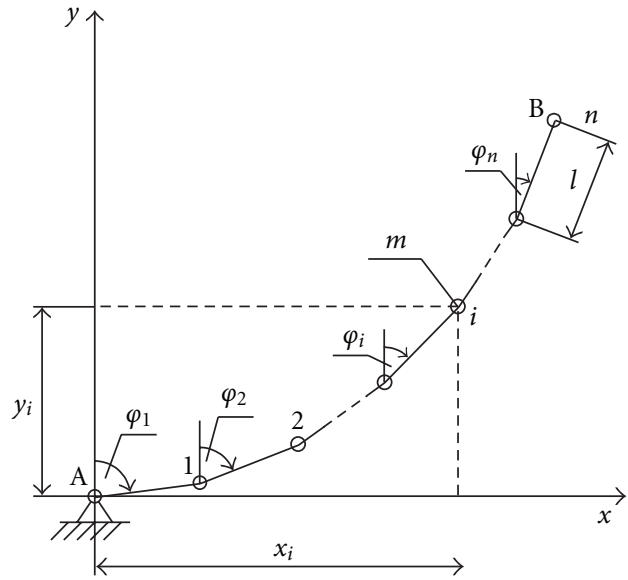

Figure 2: Discrete model in coordinate system.

Using (8), (9), and (14) in Lagrange's equations (1) and dividing by $m l^{2}$, we have

$$
\begin{aligned}
& \sum_{i=k}^{n} \sum_{j=1}^{i}\left[\cos \left(\varphi_{j}-\varphi_{k}\right) \ddot{\varphi}_{j}-\sin \left(\varphi_{j}-\varphi_{k}\right) \dot{\varphi}_{j}^{2}\right] \\
& -(n-k+1) \frac{g}{l} \sin \varphi_{k}+\frac{C}{m l^{3}} \\
& \times \sum_{i=1}^{n-1}\left[\left(\varphi_{i+1}-\varphi_{j}\right) \cdot \frac{\partial}{\partial \varphi_{k}}\left(\varphi_{i+1}-\varphi_{j}\right)\right]=0 \\
& \text { for } k=1,2, \ldots, n .
\end{aligned}
$$

Finally, it was obtained system of $n$ 2nd-order ordinary differential equations with unknown generalized coordinates $\varphi_{i}(i=1,2, \ldots, n)$. Formula (15) represents $k$ th Lagrange's equation, which describes the motion of elastica as in Figure 2.

Interpretation of boundary conditions results from formula for total potential energy of the system (6). In the presented problem, the end B of elastica is free; therefore, it is not loaded by any forces. The end A is fixed by joint; therefore, the bending moment $M=0$, and displacements in point $\mathrm{A}$ are equal zero (it has not influence on potential energy).

\section{The Lagrange's Equations in the Matrix Form}

For further analysis, the Lagrange's equations (15) were written in the matrix form. The following denotations were introduced:

$$
\boldsymbol{\Phi}=\left[\begin{array}{c}
\varphi_{1} \\
\varphi_{2} \\
\vdots \\
\varphi_{n}
\end{array}\right], \quad \frac{d \boldsymbol{\Phi}}{d t}=\dot{\boldsymbol{\Phi}}=\left[\begin{array}{c}
\dot{\varphi}_{1} \\
\dot{\varphi}_{2} \\
\vdots \\
\dot{\varphi}_{n}
\end{array}\right], \quad \frac{d^{2} \boldsymbol{\Phi}}{d t^{2}}=\ddot{\boldsymbol{\Phi}}=\left[\begin{array}{c}
\ddot{\varphi}_{1} \\
\ddot{\varphi}_{2} \\
\vdots \\
\ddot{\varphi}_{n}
\end{array}\right] .
$$

In the matrix form the Lagrange's equations (15) are

$$
\mathbf{A} \ddot{\Phi}+\mathbf{B}+\mathbf{C}+\mathbf{D}=0,
$$

where $\mathbf{A}$ is an appropriate matrix and $\mathbf{B}, \mathbf{C}, \mathbf{D}$ are vectors as follows:

$$
\mathbf{A}=\mathbf{A}(\boldsymbol{\Phi})=\left[\begin{array}{llll}
A_{11} & A_{12} & \cdots & A_{1 n} \\
A_{21} & A_{22} & \cdots & A_{2 n} \\
\cdots & \cdots & \cdots & \cdots \\
A_{n 1} & A_{n 2} & \cdots & A_{n n}
\end{array}\right]
$$

$$
\mathbf{B}=\mathbf{B}(\boldsymbol{\Phi}, \dot{\boldsymbol{\Phi}})=\left[\begin{array}{c}
B_{1} \\
B_{2} \\
\vdots \\
B_{n}
\end{array}\right] \text {, }
$$

$$
\mathbf{C}=\mathbf{C}(\boldsymbol{\Phi})=\left[\begin{array}{c}
C_{1} \\
C_{2} \\
\vdots \\
C_{n}
\end{array}\right] \text {, }
$$

$$
\mathbf{D}=\mathbf{D}(\boldsymbol{\Phi})=\left[\begin{array}{c}
D_{1} \\
D_{2} \\
\vdots \\
D_{n}
\end{array}\right] \text {. }
$$

On the basis of (15) we can write the matrix $\mathbf{A}$ and vectors $\mathrm{B}, \mathrm{C}$ and $\mathbf{D}$ as follows:

$$
\begin{gathered}
A_{k j}=S_{k j} \cos \left(\varphi_{j}-\varphi_{k}\right), \\
B_{k}=-\sum_{j=1}^{n}\left[S_{k j} \sin \left(\varphi_{j}-\varphi_{k}\right) \dot{\varphi}_{j}^{2}\right], \\
C_{k}=-(n-k+1) \frac{g}{l} \sin \varphi_{k},
\end{gathered}
$$

$D_{k}= \begin{cases}\frac{C}{m l^{3}}\left(\varphi_{1}-\varphi_{2}\right), & \text { for } k=1, \\ \frac{C}{m l^{3}}\left(-\varphi_{k-1}+2 \varphi_{k}-\varphi_{k+1}\right), & \text { for } k=2,3, \ldots, n-1, \\ \frac{C}{m l^{3}}\left(\varphi_{n}-\varphi_{n-1}\right), & \text { for } k=n,\end{cases}$ 
where the matrix $\mathbf{S}$ of dimension $n \times n$ is defined by

$$
S=\left[\begin{array}{ccccccc}
n & n-1 & n-2 & \cdots & 3 & 2 & 1 \\
n-1 & n-1 & n-2 & \cdots & 3 & 2 & 1 \\
n-2 & n-2 & n-2 & \cdots & 3 & 2 & 1 \\
\cdots & \ldots & \ldots & \cdots & \ldots & \ldots & \cdots \\
3 & 3 & 3 & \cdots & 3 & 2 & 1 \\
2 & 2 & 2 & \cdots & 2 & 2 & 1 \\
1 & 1 & 1 & \cdots & 1 & 1 & 1
\end{array}\right]
$$

The element of $k$ th row and $j$ th column of matrix $\mathbf{S}$ can be written as

$$
\begin{array}{ll}
S_{k j}=n-k+1 & \text { for } j \leq k, \\
S_{k j}=n-j+1 & \text { for } j>k .
\end{array}
$$

Its task is to realize the double summation from $i=k$ to $i=n$ and from $j=1$ to $j=i$.

Finally, it was obtained system of $n$ 2nd-order ordinary differential equations with unknown generalized coordinates $\Phi$ in matrix form

$$
\ddot{\Phi}=-\mathbf{A}^{-1}(\mathbf{B}+\mathbf{C}+\mathbf{D}) .
$$

\section{Numerical Solution of the Problem}

We can write the system of (22) in the form of system $2 n 1$ storder ordinary differential equations, introducing additional vector of generalized velocities $\dot{\boldsymbol{\Phi}}=\boldsymbol{\Omega}=\left[\begin{array}{llll}\omega_{1} & \omega_{2} & \cdots & \omega_{n}\end{array}\right]^{T}$. In this way, we obtain

$$
\begin{gathered}
\dot{\boldsymbol{\Phi}}=\mathbf{\Omega}, \\
\dot{\boldsymbol{\Omega}}=-\mathbf{A}^{-1}(\mathbf{B}+\mathrm{C}+\mathrm{D}) .
\end{gathered}
$$

The system of (23) has been solved using a standard Runge-Kutta-Fehlberg 4th-order integration method. To solve this problem numerically, the following denotations were introduced.

The vector of unknowns

$$
\mathbf{Y}=\left[\begin{array}{c}
Y_{1} \\
Y_{2} \\
\vdots \\
Y_{2 n}
\end{array}\right]
$$

where

$$
\begin{gathered}
Y_{i}=\varphi_{i} \text { for } i=1,2, \ldots, n, \\
Y_{i}=\omega_{i-n} \text { for } i=n+1, n+2, \ldots, 2 n .
\end{gathered}
$$

The right side of (22) has been denoted by vector F; that is,

$$
\mathbf{F}=-\mathbf{A}^{-1}(\mathbf{B}+\mathbf{C}+\mathbf{D}), \quad \mathbf{F}=\left[\begin{array}{c}
F_{1} \\
F_{2} \\
\vdots \\
F_{n}
\end{array}\right]
$$

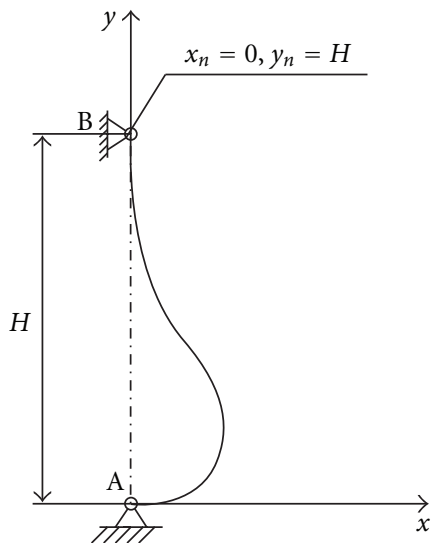

Figure 3: The elastica with two ends fixed at points A and B.

Finally, the following system of differential equations was solving:

$$
\begin{gathered}
\dot{Y}_{1}=Y_{n+1}, \\
\vdots \\
\dot{Y}_{n}=Y_{2 n}, \\
\dot{Y}_{n+1}=F_{1}, \\
\vdots \\
\dot{Y}_{2 n}=F_{n},
\end{gathered}
$$

with initial conditions for $t=t_{0}$

$$
\boldsymbol{\Phi}=\boldsymbol{\Phi}^{0}=\left[\begin{array}{c}
\varphi_{1}^{0} \\
\vdots \\
\varphi_{n}^{0}
\end{array}\right], \quad \boldsymbol{\Omega}=\boldsymbol{\Omega}^{0}=\left[\begin{array}{c}
\omega_{1}^{0} \\
\vdots \\
\omega_{n}^{0}
\end{array}\right]
$$

\section{The Lagrange's Equations with Constraints}

In a previous section the elastica fixed only at point $\mathrm{A}$ was considered. The other end B of elastica was free (Figure 2). In this case, generalized coordinates $\varphi_{k}$ were completely independent in themselves. In many problems connected with the motion of elastic, there are some constraints concerning angles $\varphi_{k}$. Then, the Lagrange's equations undergo modification.

In this section the motion of elastica with two ends fixed at two points A and B using joints as in Figure 3 will be considered. This case may be used for example to simulate fabric folding and so on. Then, the boundary conditions for the end B (which previously was free) are as follows:

$$
\begin{gathered}
x_{n}=0, \\
y_{n}-H=0 .
\end{gathered}
$$


In accordance with (2) for $x_{n}$ and $y_{n}$ we can rewrite the following two conditions of constraints (29) obtaining

$$
\begin{gathered}
f_{1} \equiv l \sum_{k=1}^{n} \sin \varphi_{k}=0, \\
f_{2} \equiv l \sum_{k=1}^{n} \cos \varphi_{k}-H=0 .
\end{gathered}
$$

Thus, we can write the Lagrange's equations in the following form:

$$
\begin{array}{r}
\frac{d}{d t}\left(\frac{\partial T}{\partial \dot{\varphi}_{k}}\right)-\frac{\partial T}{\partial \varphi_{k}}+\frac{\partial U}{\partial \varphi_{k}}+\lambda_{1} \frac{\partial f_{1}}{\partial \varphi_{k}}+\lambda_{2} \frac{\partial f_{2}}{\partial \varphi_{k}}=0 \\
k=1,2, \ldots, n
\end{array}
$$

where $\lambda_{1}, \lambda_{2}$ are the unknown Lagrange's multipliers (their interpretations are the appropriate reaction forces in point B-horizontal and vertical).

Derivatives $\partial f_{1} / \partial \varphi_{k}$ and $\partial f_{2} / \partial \varphi_{k}$ are as below:

$$
\begin{gathered}
\frac{\partial f_{1}}{\partial \varphi_{k}}=l \cos \varphi_{k}, \\
\frac{\partial f_{2}}{\partial \varphi_{k}}=-l \sin \varphi_{k} .
\end{gathered}
$$

Let right side of the $k$ th (15) be denoted by $\Psi_{k}(\varphi, \dot{\varphi})$. Therefore, using (31) and (32), we have the following system of equations:

$$
\Psi_{k}(\varphi, \dot{\varphi})+\frac{\lambda_{1}}{m l} \cos \varphi_{k}-\frac{\lambda_{2}}{m l} \sin \varphi_{k}=0, \quad k=1,2, \ldots, n .
$$

Using matrix notation, we can write new matrix form of the system (33)

$$
\mathbf{A} \ddot{\Phi}+\mathbf{B}+\mathbf{C}+\mathbf{D}+\lambda_{1} \cdot \mathbf{E}+\lambda_{2} \cdot \mathbf{G}=0,
$$

where

$$
\begin{gathered}
\mathbf{E}=\mathbf{E}(\boldsymbol{\Phi})=\left[\begin{array}{c}
E_{1} \\
E_{2} \\
\vdots \\
E_{n}
\end{array}\right], \quad E_{k}=\frac{\cos \varphi_{k}}{m l}, \quad k=1,2, \ldots, n, \\
\mathbf{G}=\mathbf{G}(\boldsymbol{\Phi})=\left[\begin{array}{c}
G_{1} \\
G_{2} \\
\vdots \\
G_{n}
\end{array}\right], \quad G_{k}=-\frac{\sin \varphi_{k}}{m l}, \quad k=1,2, \ldots, n .
\end{gathered}
$$

The system of (34) with the (30) presents the system of $n+2$ differential-algebraic equations with $n+2$ unknowns, that is, $n$ generalized coordinates $\varphi_{i}(i=1,2, \ldots, n)$ and two
Lagrange's multipliers $\lambda_{1}, \lambda_{2}$. To solve such problem, (30) have been differentiated two times in respect of time

$$
\begin{gathered}
\sum_{k=1}^{n}\left(\cos \varphi_{k} \cdot \ddot{\varphi}_{k}-\sin \varphi_{k} \cdot \dot{\varphi}_{k}^{2}\right)=0, \\
\sum_{k=1}^{n}\left(-\sin \varphi_{k} \cdot \ddot{\varphi}_{k}-\cos \varphi_{k} \cdot \dot{\varphi}_{k}^{2}\right)=0 .
\end{gathered}
$$

Next, from (34), the multipliers $\lambda_{1}$ and $\lambda_{2}$ have been eliminated successively, using twice Gaussian elimination. After this, (34) together with (36) present the system of $n$ 2nd-order ordinary differential equations in respect of $\varphi_{i}(i=1,2, \ldots, n)$. Using matrix notation, we have

$$
\widehat{\mathbf{A}} \ddot{\boldsymbol{\Phi}}+\widehat{\mathbf{B}}+\widehat{\mathbf{C}}+\widehat{\mathbf{D}}=0
$$

similarly as (17) in Section 3. The components of new matrix $\widehat{\mathbf{A}}$ and vectors $\widehat{\mathbf{B}}, \widehat{\mathbf{C}}$ i $\widehat{\mathbf{D}}$ are as below:

$$
\text { For } k=1,2, \ldots, n-2
$$

$$
\begin{aligned}
& \widehat{A}_{k j}=\left(A_{k j} \cos \varphi_{k+1}-A_{k+1 j} \cos \varphi_{k}\right) \sin \left(\varphi_{k+1}-\varphi_{k+2}\right) \\
& -\left(A_{k+1 j} \cos \varphi_{k+2}-A_{k+2 j} \cos \varphi_{k+1}\right) \\
& \times \sin \left(\varphi_{k}-\varphi_{k+1}\right), \quad \text { for } j=1,2, \ldots, n, \\
& \widehat{B}_{k}=\left(B_{k} \cos \varphi_{k+1}-B_{k+1} \cos \varphi_{k}\right) \sin \left(\varphi_{k+1}-\varphi_{k+2}\right) \\
& -\left(B_{k+1} \cos \varphi_{k+2}-B_{k+2} \cos \varphi_{k+1}\right) \sin \left(\varphi_{k}-\varphi_{k+1}\right), \\
& \hat{C}_{k}=\left(C_{k} \cos \varphi_{k+1}-C_{k+1} \cos \varphi_{k}\right) \sin \left(\varphi_{k+1}-\varphi_{k+2}\right) \\
& -\left(C_{k+1} \cos \varphi_{k+2}-C_{k+2} \cos \varphi_{k+1}\right) \sin \left(\varphi_{k}-\varphi_{k+1}\right), \\
& \hat{D}_{k}=\left(D_{k} \cos \varphi_{k+1}-D_{k+1} \cos \varphi_{k}\right) \sin \left(\varphi_{k+1}-\varphi_{k+2}\right) \\
& -\left(D_{k+1} \cos \varphi_{k+2}-D_{k+2} \cos \varphi_{k+1}\right) \sin \left(\varphi_{k}-\varphi_{k+1}\right), \\
& \widehat{A}_{n-1 j}=\cos \varphi_{j} \text {, } \\
& \widehat{A}_{n j}=-\sin \varphi_{j}, \quad \text { for } j=1,2, \ldots, n \text {, } \\
& \widehat{B}_{n-1}=-\sum_{j=1}^{n}\left(\sin \varphi_{j} \cdot \dot{\varphi}_{j}^{2}\right), \\
& \widehat{B}_{n}=-\sum_{j=1}^{n}\left(\cos \varphi_{j} \cdot \dot{\varphi}_{j}^{2}\right), \\
& \widehat{C}_{n-1}=\widehat{C}_{n}=0, \\
& \widehat{D}_{n-1}=\widehat{D}_{n}=0 \text {. }
\end{aligned}
$$

The system of (37) has been transformed to the form similarly as in Section 5; that is,

$$
\ddot{\Phi}=-\widehat{\mathbf{A}}^{-1}(\widehat{\mathbf{B}}+\widehat{\mathbf{C}}+\widehat{\mathbf{D}}) .
$$



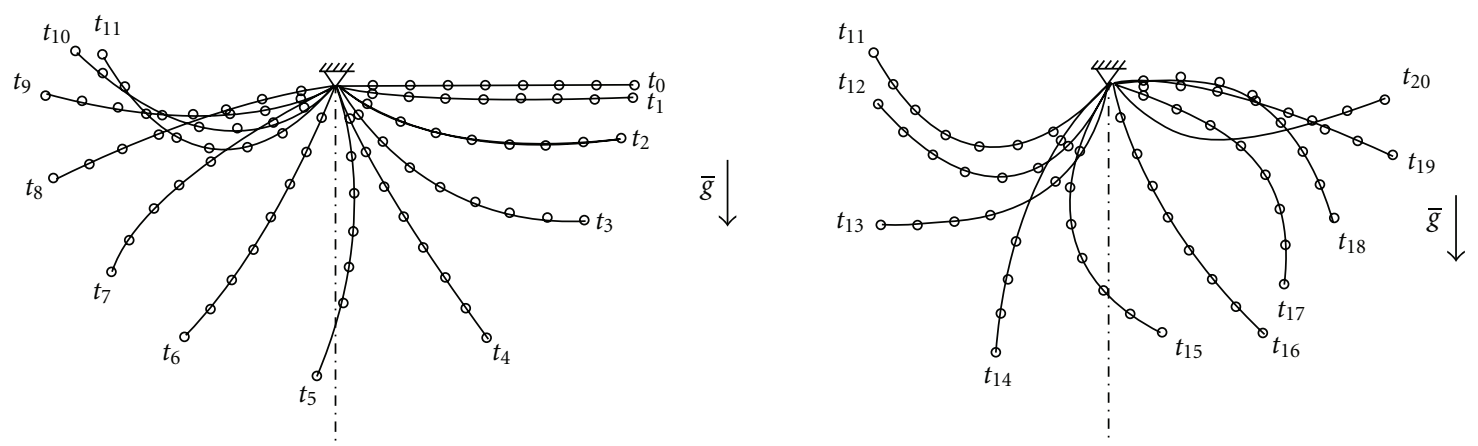

FIgURE 4: The shape of elastica in the following time instant from $t_{0}$ to $t_{20}$.

Next, we can write

$$
\begin{gathered}
\dot{\Phi}=\Omega \\
\dot{\Omega}=-\widehat{\mathbf{A}}^{-1}(\widehat{\mathbf{B}}+\widehat{\mathbf{C}}+\hat{\mathbf{D}}) .
\end{gathered}
$$

Finally, the system of (40) has been solved using a standard Runge-Kutta-Fehlberg 4th-order integration method.

The considerations presented above concerned with the elastica for which the end B was fixed by joint. Of course, the other conditions of constraints may be introduced for this one.

If we want to allow vertical motion of the end $\mathrm{B}$, then we have only one constraint for coordinate $x$ of the end B : $x$ must be always equal to value $a=$ const., and we have only one algebraic equation (conditions of constraints) in the form of

$$
f_{1} \equiv x_{n}-a=0
$$

The Lagrange's equations are simple in this case, because we have only one Lagrange's multipliers $\lambda_{1}$.

If we want to have the end $B$ fixed at point $B$ at an angle of $\varphi=\alpha$, where $x=a, y=b$, then there are three conditions of constraints

$$
\begin{aligned}
& f_{1} \equiv x_{n}-a=0, \\
& f_{2} \equiv y_{n}-b=0, \\
& f_{3} \equiv \varphi_{n}-\alpha=0,
\end{aligned}
$$

and three Lagrange's multipliers $\lambda_{1}, \lambda_{2}$, and $\lambda_{3}$.

\section{The Results of Calculations}

To present the results of calculations and accuracy of numerical method two numerical cases were considered.

The first one concerned the motion of heavy elastica under the influence of the gravity force in conservative force field. The elastica was fixed byjoint at one end only as in Figure 2.

In the second one, the elastica was fixed by joint at two ends as in Figure 3.
6.1. The Motion of the Elastica Fixed at One Point. In this case, the elastica fixed at one point by joint as in Figure 2 will be considered. The second end is free. The motion is under the influence of the gravity and elastic forces.

The properties of the elastica are as follows:

(i) bending rigidity, $C=0.0025 \mathrm{Nm}^{2}$,

(ii) linear mass density, $\rho=0.01 \mathrm{~kg} / \mathrm{m}$,

(iii) length, $L=1 \mathrm{~m}$.

Too many points $n$ of division can result in long time of calculations. In the example below, it has been assumed that $n=8$.

The initial conditions (28) are as follows:

$$
\begin{aligned}
\varphi_{k}^{0} & =\frac{\pi}{2} \mathrm{rad}, \quad \text { for } k=1,2, \ldots, n . \\
\omega_{k}^{0} & =0 \mathrm{rad} / \mathrm{s},
\end{aligned}
$$

In the initial instant, the elastica has rectilinear, horizontal shape. The velocity of all points is zero. After solving the (23) with the time step $\Delta t=0.005 \mathrm{~s}$, we have obtained the shape of elastica in the following time instant $t_{0}, t_{1}, t_{2}, \ldots$ The shapes are presented in Figure 4. Figure 5 shows variations of generalized coordinates $\varphi_{1}, \varphi_{2}, \ldots, \varphi_{8}$ in the respect of time $t$. Figure 6 shows variations of generalized coordinates $\varphi_{1}, \varphi_{2}, \ldots, \varphi_{8}$ in the respect of time $t$ for elastica of bending rigidity 10 times less than previously $\left(C=0.00025 \mathrm{Nm}^{2}\right)$.

In Figures 5 and 6, the heavy dashed line represents graph of angular displacement of rigid rod (physical pendulum) fixed in the same point as elastica (Figure 7).

From the graphs, it may be inferred that in the initial state of the motion the points of elastica oscillate around the dynamic equilibrium (as for physical pendulum). The rigid rod (Figure 7) can be treated as the elastica of bending rigidity $C=\infty$ for which all points have permanently the same angular displacements $\varphi_{k}=\varphi$ dla $k=1,2, \ldots, n$. As the time goes, deviations from dynamic equilibrium become bigger and bigger. below.

In order to check accuracy of calculations, we follow as

For chosen coordinate $\varphi_{8}$, its values marked by $\varphi_{8}^{\Delta t_{1}}$ with given time step $\Delta t_{1}=0.005 \mathrm{~s}$ have been calculated. Next, the calculations have been repeated for the less time step 


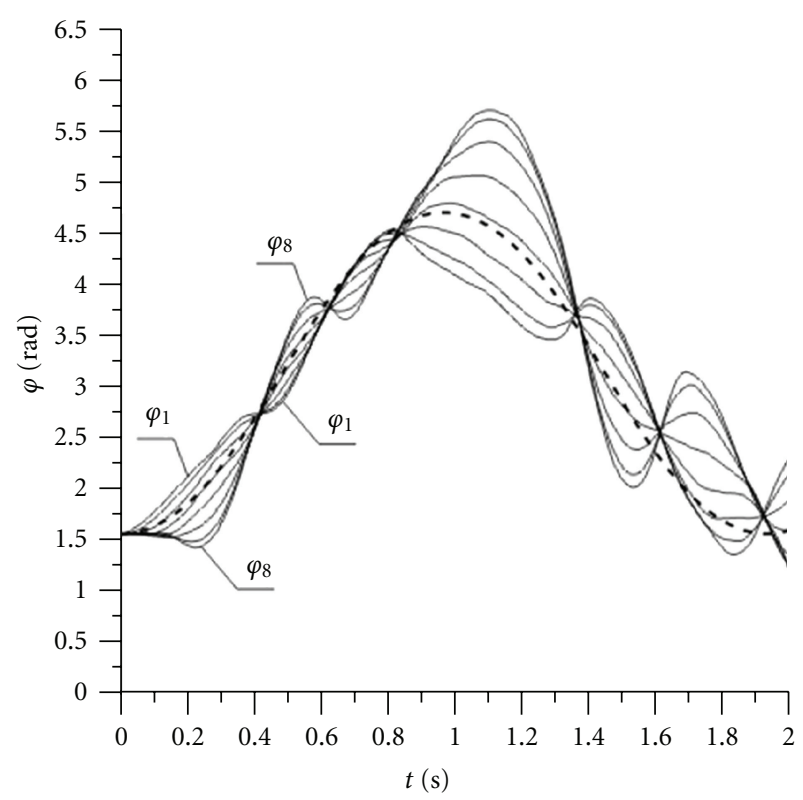

FIGURE 5: The variation of generalized coordinates $\varphi_{1}, \varphi_{2}, \ldots, \varphi_{8}$ in the respect of time $t$.

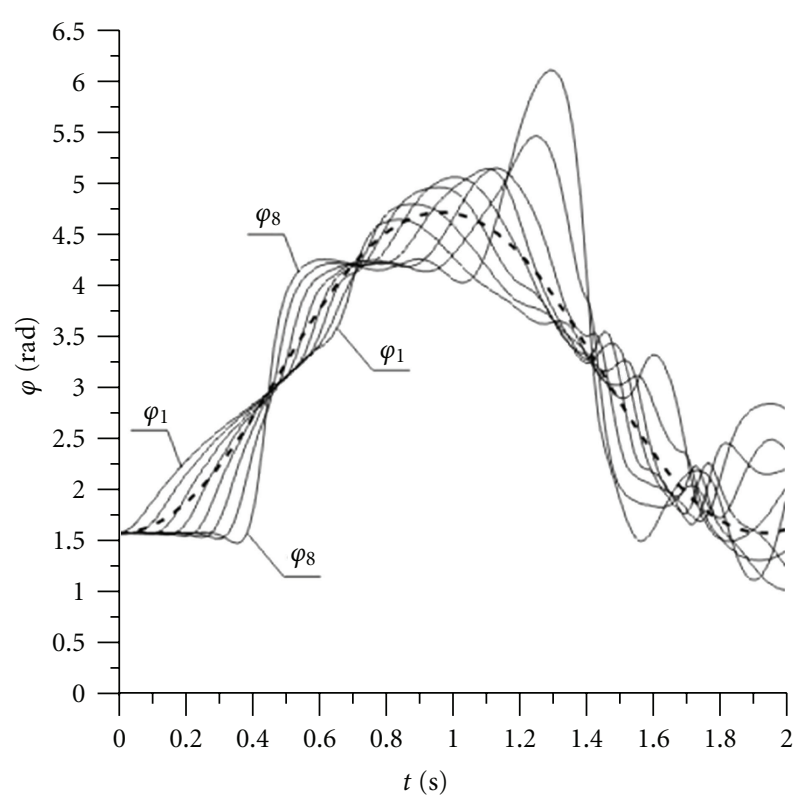

FIGURE 6: The variation of generalized coordinates $\varphi_{1}, \varphi_{2}, \ldots, \varphi_{8}$ in the respect of time $t$ for bending rigidity of $C=0.00025 \mathrm{Nm}^{2}$.

$\Delta t_{2}=1 / 2 \Delta t_{1}$. The difference $\Delta \varphi_{8}=\varphi_{8}^{\Delta t_{1}}-\varphi_{8}^{\Delta t_{2}}$ has been determined for following $t_{j}=t_{0}+i \cdot \Delta t_{1}$.

The calculations have been made for $C=0.0025 \mathrm{Nm}^{2}$. The graph of differences $\Delta \varphi_{8}$ for following time instant is presented in Figure 8.

The differences $\Delta \varphi_{8}$ are not big at all (between -0.00019 and $0.00022 \mathrm{rad})$, even after large number of iterations $(i=$ 400). The accuracy turned out to be satisfactory.

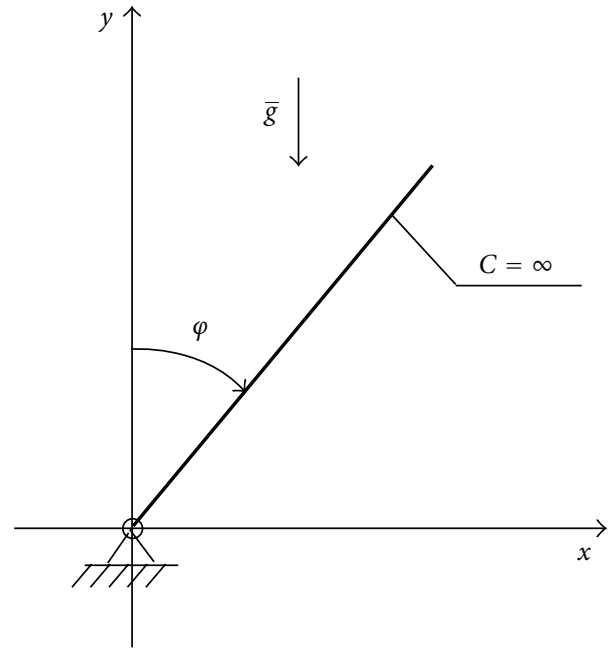

FIGURE 7: The rigid rod as physical pendulum.

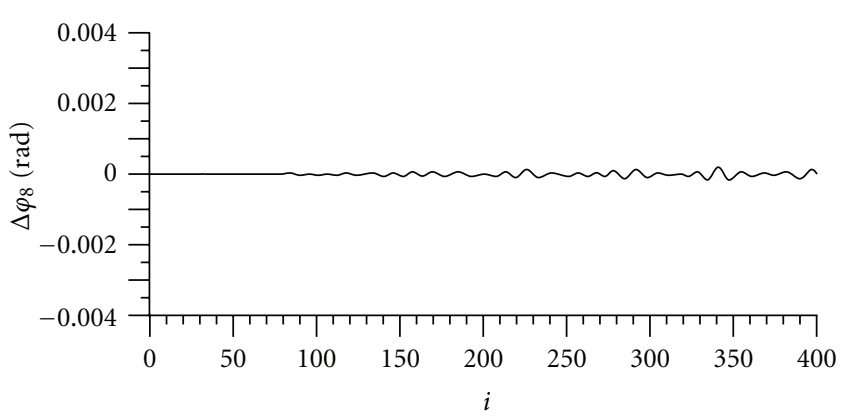

Figure 8: Test of accuracy of generalized coordinate $\varphi_{8}$ in the following time instant.

6.2. The Motion of the Elastica Fixed at Two Points. In this case, the elastica fixed at two points by joints as in Figure 3 will be considered. The motion is under the influence of the gravity and elastic forces.

The properties of the elastica are as follows:

(i) bending rigidity, $C=0.0015 \mathrm{Nm}^{2}$,

(ii) linear mass density, $\rho=0.01 \mathrm{~kg} / \mathrm{m}$,

(iii) length, $L=1.2 \mathrm{~m}$.

Number of points of division is $n=16$. The system of (40) has been solved using Runge- Kutta-Fehlberg 4th-order integration method with time step $\Delta t=0,005 \mathrm{~s}$. The initial conditions (28) are as follows:

$$
\begin{aligned}
& \varphi_{k}^{0}=\frac{\pi}{2}\left[1-\frac{2(k-1)}{n-1}\right] \mathrm{rad}, \quad \text { for } k=1,2, \ldots, n . \\
& \omega_{k}^{0}=0 \mathrm{rad} / \mathrm{s},
\end{aligned}
$$

Finally, the shape of the elastica in the following time instant $t_{j}$ has been obtained. It is present in Figure 9.

In order to check accuracy of calculations, we tested how the boundary conditions (29) change as time goes by. In this 


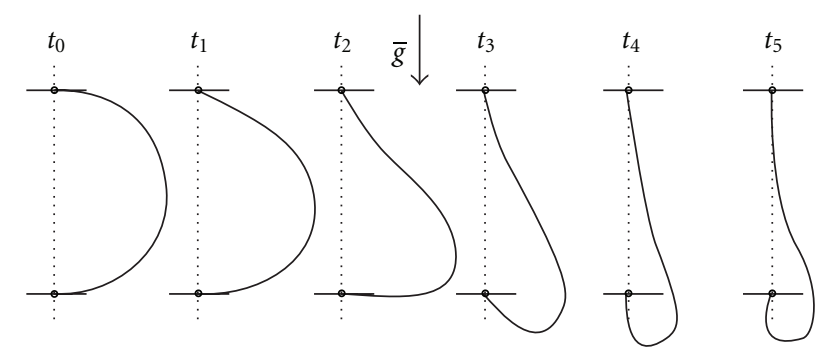

Figure 9: The shape of the elastica in the following time instant.

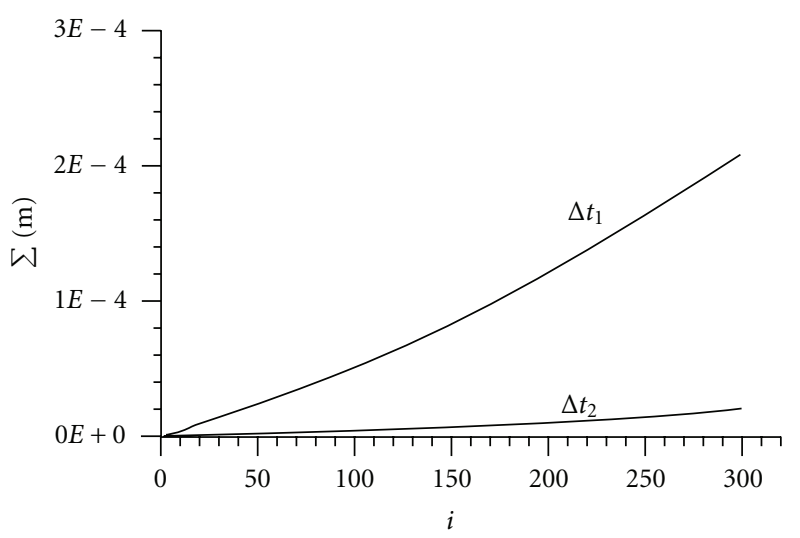

FIGURE 10: The analysis of fulfillment of the boundary conditions $\sum=\left|f_{1}\right|+\left|f_{2}\right|$.

way, we can check how the algorithm secures fulfillment of the boundary conditions

$$
\begin{gathered}
f_{1} \equiv x_{n}=0, \\
f_{2} \equiv y_{n}-H=0,
\end{gathered}
$$

As a measure of deviation of boundary conditions, we can take $\Sigma=\left|f_{1}\right|+\left|f_{2}\right|$, which, if the boundary conditions are fulfilled, should be equal zero. The calculations have been made for two time steps: $\Delta t_{1}=0.005 \mathrm{~s}$ i $\Delta t_{2}=1 / 2 \Delta t_{1}$. The variation of $\sum$ in respect of number of iterations are presented in Figure 10 for two time steps.

From Figure 10, we can conclude that for two times less time step deviation of boundary conditions was significant less. For $\Delta t_{1}$ after 300 iterations, deviation was about 2 . $10^{-4} \mathrm{~m}$, and for $\Delta t_{2}=1 / 2 \Delta t_{1}$, deviation was about $2 \cdot 10^{-5} \mathrm{~m}$ (10 times less).

\section{Conclusion}

After carrying out series of numerical tests, it can be concluded that Lagrange's equations are practically useful for the analysis of the motion of heavy elastica. It can be used for simulation of many problems from the field of textile mechanics, for example, fabric folding (Figure 11), motion of the sewing thread and so on. Similar investigations, but not using Lagrange's equations, have been described by Lloyd in [7].

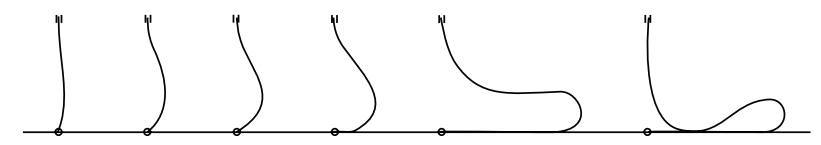

FIgURE 11: The simulation of fabric folding using Lagrange's equations.

The results of calculations show clearly wave nature of phenomenon. The bending rigidity has not influence on the convergence of the results. The analyzed motion of elastica is stable owing to initial conditions.

\section{References}

[1] P. Szablewski and W. Kobza, "Numerical analysis of Peirce's Cantilever test for the bending rigidity of textiles," Fibres and Textiles in Eastern Europe, vol. 11, no. 4, pp. 54-57, 2003.

[2] J. Leyko, Dynamika Układów Materialnych, vol. II, PWN, Warszawa, Poland, 1961.

[3] W. Greiner, Classical mechanics: Systems of Particles and Hamiltonian Dynamics, Springer, New York, NY, USA, 2003.

[4] O.M. O’Reilly, Intermediate Dynamics for Engineers: A Unified Treatment of Newton Euler and Lagrangian Mechanics, Cambridge University Press, New York, NY, USA, 2008.

[5] J. R. Taylor, Classical Mechanics, University Science Books, Mill Valley, Calif, USA, 2005.

[6] P. Szablewski, "Modelling of dynamical behaviour of flat textile structure using lagrange's equations," in Proceedings of the 10th International Conference (IMTEX '09), pp. 35-38, Łódź, Poland, 2009.

[7] D. W. Lloyd, W. J. Shanahan, and M. Konopasek, "The folding of heavy fabric sheets," International Journal of Mechanical Sciences, vol. 20, no. 8, pp. 521-527, 1978. 

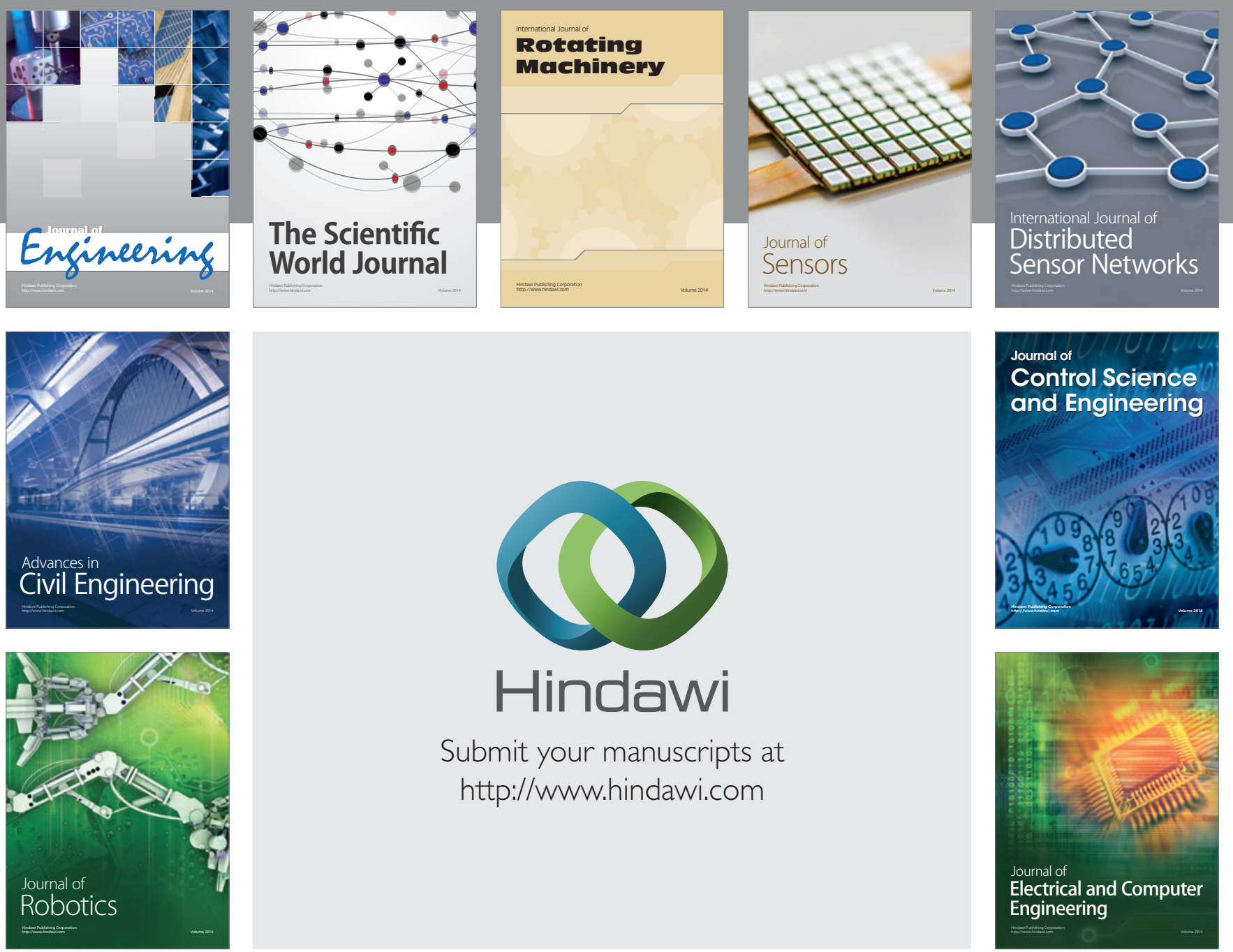

Submit your manuscripts at

http://www.hindawi.com
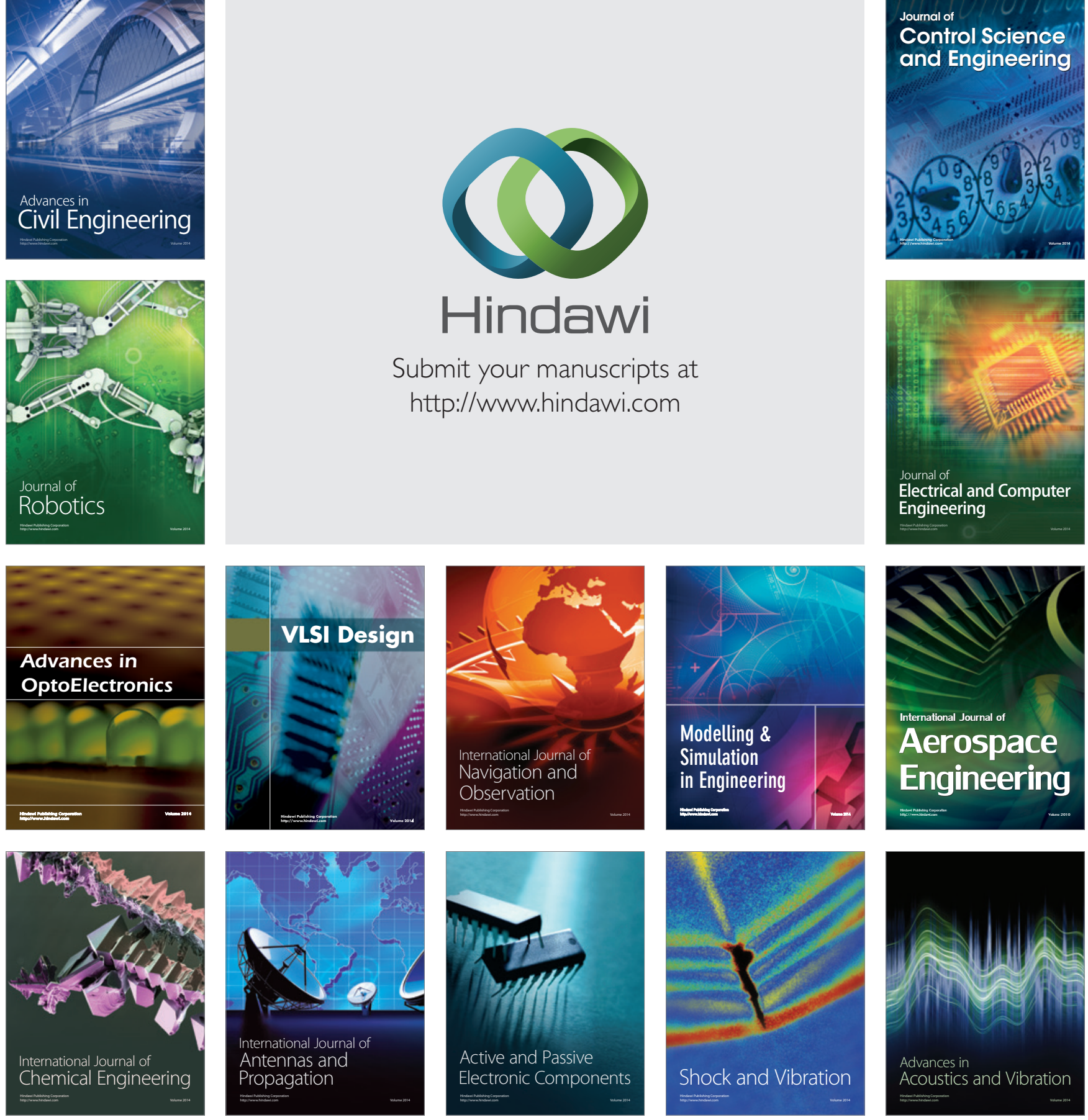